\title{
Acute promyelocytic leukemia in a child with reticulin fibrosis
}

\author{
Tekin Aksu $^{1}$ (D) $\cdot$ Barış Kuşkonmaz $^{1}$ (i) $\cdot$ Sule Unal ${ }^{1}$ (D) $\cdot$ Arzu Saglam $^{2}$ (D) Fatma Gümrük $^{1}$ (D)
}

Received: 16 June 2020 / Accepted: 15 July 2020 / Published online: 22 July 2020

(C) Springer-Verlag GmbH Germany, part of Springer Nature 2020

\begin{abstract}
Reactive myelofibrosis (MF) may be associated with infectious, inflammatory, or neoplastic conditions. Extensive myelofibrosis and indolent course are frequent in acute megakaryoblastic leukemia, but these findings are rarely reported in acute promyelocytic leukemia (APL). Patients with neoplastic disorders associated with MF are reported to have an inferior outcome. However, the effect of this association is not apparent in APL patients. This report presents a case of APL in a pediatric patient in association with significant reticulin fibrosis and osteosclerosis. The case highlights that fibrosis in the marrow can make it more challenging to diagnose acute leukemia. The $P M L-R A R A$ transcripts can be present with subtle clinical or hematological findings, emphasizing the importance of molecular studies in unexplained cytopenias. Furthermore, in the case of APL, as opposed to other myeloid neoplasms, fibrosis does not appear to be associated with a worse prognosis and can resolve after treatment.
\end{abstract}

Keywords Acute promyelocytic leukemia $\cdot$ Myelofibrosis $\cdot$ Osteosclerosis $\cdot$ Children

\section{Introduction}

Reactive myelofibrosis (MF) may be associated with infectious, inflammatory, or neoplastic conditions, such as Epstein-Barr virus (EBV), systemic lupus erythematosus, myelodysplastic syndrome (MDS) or myeloproliferative neoplasms, acute lymphoblastic leukemia (ALL), and acute myeloid leukemia (AML) [1]. Extensive myelofibrosis and indolent course are frequent in acute megakaryoblastic leukemia subtype, but these findings are rarely reported in acute

Tekin Aksu

tekinaksu@gmail.com

Barıș Kuşkonmaz

bkuskonmaz@gmail.com

Sule Unal

suleunal@hacettepe.edu.tr

Arzu Saglam

eminearzusaglam@gmail.com

Fatma Gümrük

fgumruk@hacettepe.edu.tr

1 Department of Pediatrics, Division of Hematology, Hacettepe University Faculty of Medicine, Sihhiye, 06100 Ankara, Turkey

2 Department of Pathology, Hacettepe University Faculty of Medicine, Ankara, Turkey promyelocytic leukemia (APL) [2]. The real incidence of MF in childhood acute leukemias is hard to estimate because, in most cases, bone marrow aspirate is sufficient to establish the diagnosis and bone marrow biopsy is not performed.

Primary MF is characterized by anemia, extramedullary hematopoiesis, and immature myeloid and erythroid precursors, teardrop-shaped red cells on the peripheral blood smear. Also, it is defined by progressive reticulin and collagen deposition in the bone marrow leading to fibrosis. Acute myeloid leukemia subtypes with fibrotic marrow, presenting with pancytopenia and mild splenomegaly, may mislead to a diagnosis of primary MF [2]. This report presents a case of APL in a pediatric patient in association with significant reticulin fibrosis and osteosclerosis.

\section{Clinical history}

A $5^{1 / 2}$-year-old girl presented with easy bruising, fatigue, weakness, and cytopenias. There were bruises around the knees and elbows on her physical exam; no splenomegaly or hepatomegaly was present. The medical history revealed an anal atresia surgery when she was 9 months old and diagnosed with immunoglobulin A deficiency since the age of 3. Family history was unremarkable, and there was no consanguinity between parents. Laboratory studies of the patient at presentation showed hemoglobin $12.7 \mathrm{~g} / \mathrm{dL}$ (lower limit 11.5), mean corpuscular volume $77.7 \mathrm{fL}$ (lower limit 75), platelets $97 \times$ 
$10^{9} / \mathrm{L}(150-450)$, total leukocytes $3.5 \times 10^{9} / \mathrm{L}(5-14.5)$, neutrophils $1.1 \times 10^{9} / \mathrm{L}(1.5-8)$, and a reticulocyte count was $1.16 \%$. No immature cells, blasts, or tear-drop-shaped cells were present on the peripheral blood smear. Initial workup for cytopenias revealed the presence of the parvovirus B19, which was quantified as $1995 \mathrm{IU} / \mathrm{mL}\left(100-100 \times 10^{6}\right)$ in the serum by PCR analysis and managed with $2 \mathrm{~g} / \mathrm{kg}$ human normal immunoglobulin for intravenous use (IVIG). Bone marrow (BM) aspiration was performed 1 week after the admission due to the persistence of cytopenias. It disclosed a normocellular marrow with erythroid hypoplasia with few mature normoblasts, no blastic infiltration, and no giant erythroblasts suggestive of harboring parvovirus inclusions. However, a few atypical densely granulated cells suspicious for atypical promyelocytes with coarse and irregular granulations were noted. Concurrently, she developed hypofibrinogenemia without signs of disseminated intravascular coagulation and was treated with fresh frozen plasma. The cytogenetic studies of this initial BM did not display acquired clonal chromosomal aberrations. The diepoxybutane analysis was performed to exclude Fanconi anemia and revealed no breakage, and hemoglobin F level was normal for age. Also, the flow cytometric analysis of the BM did not disclose a discernible atypical population. However, 3 weeks later, during the follow-up visit, results of the molecular studies of the initial BM were received and showed 2061 copies of $P M L-R A R A / 10^{4} A B L 1$ of bcr1 isoform by Q-RT-PCR. At this visit, blood count revealed hemoglobin $9.9 \mathrm{~g} / \mathrm{dL}$, total leukocytes $2.7 \times 10^{9} / \mathrm{L}$, and platelets $9 \times 10^{9} / \mathrm{L}$. A peripheral blood smear showed immature myeloid cells characterized by abundant red to purple cytoplasmic granules numerous enough to obscure the cytoplasmic borders. Serum biochemistry, including renal function tests, was normal except for lactate dehydrogenase level 393 U/L (110-295). A second BM aspiration and biopsy were performed, revealing $62 \%$ abnormal promyelocytes, which contained abundant dysplastic granules and Auer rods, suggesting APL in a medium cellularity bone marrow (Fig. 1). Flow cytometric evaluation of the bone marrow shows $56 \%$ atypical cell population with high side scatter characteristics, strongly suggestive of APL, expressing CD13/CD33/CD117/myeloperoxidase (MPO), lacking CD34/HLA-DR (Fig. 2). The second review of the flow cytometric evaluation of the first aspirate showed no atypical cells. H\&E sections of the biopsy disclosed reticulin fibrosis grade III/III and osteosclerotic bone trabeculae, along with a small focus of immature cells (Fig. 3). At high power magnification, these immature cells were characterized by eccentric blastic nuclei, abundant granulated cytoplasm, and MPO positivity on immunohistochemistry (Fig. 4). The accompanying aspirate smear that was sent to the pathology laboratory lacked particles and was extremely hypocellular; only a few blastic cells, some with bilobed nuclei consistent with APL, could be identified among the small number of cells present (Fig. 4

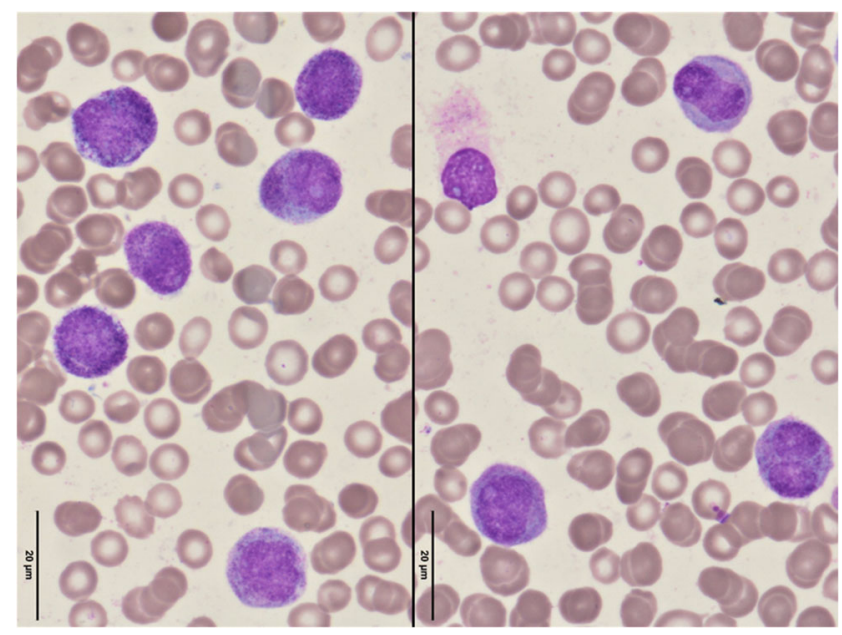

Fig. 1 Photomicrographs of the bone marrow aspirate. Promyelocytes with abundant azurophilic granules can be seen; granules are irregular looking. It also noted reniform and bilobed nuclear contours

inset). Cytogenetic analysis of this second BM showed a karyotype of 46XX (20 metaphase cells), and fluorescence in situ hybridization (FISH) for $\mathrm{t}(15 ; 17)$ (q22;q21) (200 interphase cells) was negative. PCR analysis revealed 1707 copies of $P M L-R A R A / 10^{4} A B L 1$ of bcr1 isoform in this second $\mathrm{BM}$, confirming the diagnosis of APL. No evidence of an accompanying disease that could explain the presence of osteosclerosis could be found. After the diagnosis of APL, all-trans retinoic acid (ATRA) was commenced with induction chemotherapy. Ten days after ATRA treatment, she developed tachypnea, lung infiltrates, and weight gain, which prompted a diagnosis of ATRA differentiation syndrome, and this recovered with dexamethasone administration. After the first chemotherapy course of BFM-AML-2013 protocol, she achieved morphological remission, which includes cytarabine, idarubicin, etoposide, and ATRA. Four months after the diagnosis, she completed her chemotherapy regimen, and reticulin fibrosis disappeared (Fig. 5). Also, the PML-RARA transcript was found to be negative.

\section{Discussion}

Acute promyelocytic leukemia accounts for $5-10 \%$ of pediatric AML and occurs equally among all age groups [3]. In Turkey, a study of 83 childhood AML patients revealed a $20.5 \%$ incidence of APL with a median age of 13.5 [4]. Bone marrow fibrosis and osteosclerosis are unusual in childhood APL, which creates a diagnostic challenge. The aspirates may not reveal adequate cellular elements, hindering diagnosis, necessitating biopsy, and molecular workup might be more crucial in the diagnosis. About 5\% of the APL cases are negative for $\mathrm{t}(15 ; 17)$, which is the APL's cytogenetic hallmark. However, the presence of PML-RARA transcripts is more specific than $\mathrm{t}(15 ; 17)$ [3]. The presented case showed 

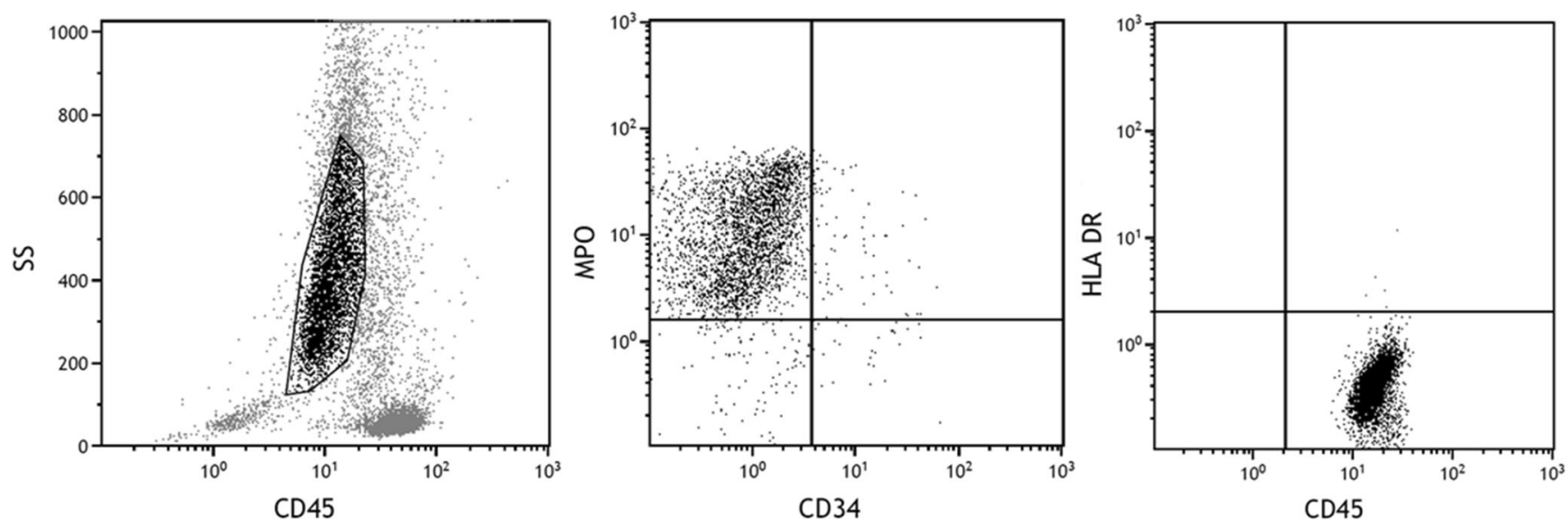

Fig. 2 Flow cytometric evaluation of the bone marrow shows a significant cell population with high side scatter characteristics (left panel), strongly suggestive of APL, positive with MPO and lacks CD34 (middle panel), and lacks HLA-DR (right panel)

PML-RARA transcripts, before any sign of APL on flow cytometry or microscopic examinations of MGG-stained slides of the initial aspirate. Since the patient's hypofibrinogenemia could not be explained by another etiology, looking back can be accepted as an early warning sign of APL stressing the importance of close monitoring of such patients.

Bone marrow consists of essential structural fibrils like reticulin and collagen. These may be increased in various neoplastic or non-neoplastic disorders. Increased reticulin or collagen fibrils represent a bone marrow abnormality. Unlike reticulin, collagen deposition may not be reversible [1]. The pathophysiology of myelofibrosis is not precise, but it is suggested to be the result of an altered hematopoietic activity.
Recent studies have demonstrated that MF is associated with increased cytokines or growth factors secreted by megakaryocytes and platelets, such as platelet-derived growth factor and transforming growth factor- $\beta$ (TGF- $\beta$ ) [1]. Additionally, leukemic cells expressing CD34 and HLA-DR may contribute to reticulin synthesis [5]. However, APL cases usually do not express CD34 or HLA-DR. Abou Dalle et al. hypothesized that lack of CD34 or HLA-DR expression may explain the low incidence of myelofibrosis in APL cases [5]. Mori et al. described an APL patient with overexpression of TGF- $\beta$ in his leukemic cells associated with MF. However, at the time of relapse, neither increase in expression of TGF- $\beta$ nor MF was observed [6]. Unfortunately, we were not able to study these
Fig. 3 Photomicrographs of the bone marrow biopsy. Marrow space shows prominent fibrosis, bony trabeculae $(*)$ are sclerotic with irregular borders and thickening, a relatively cellular area $(* *)$ composed of mononucleated cells can be seen (A). Reticulin stain highlights the severity of reticulin fibrosis (B)

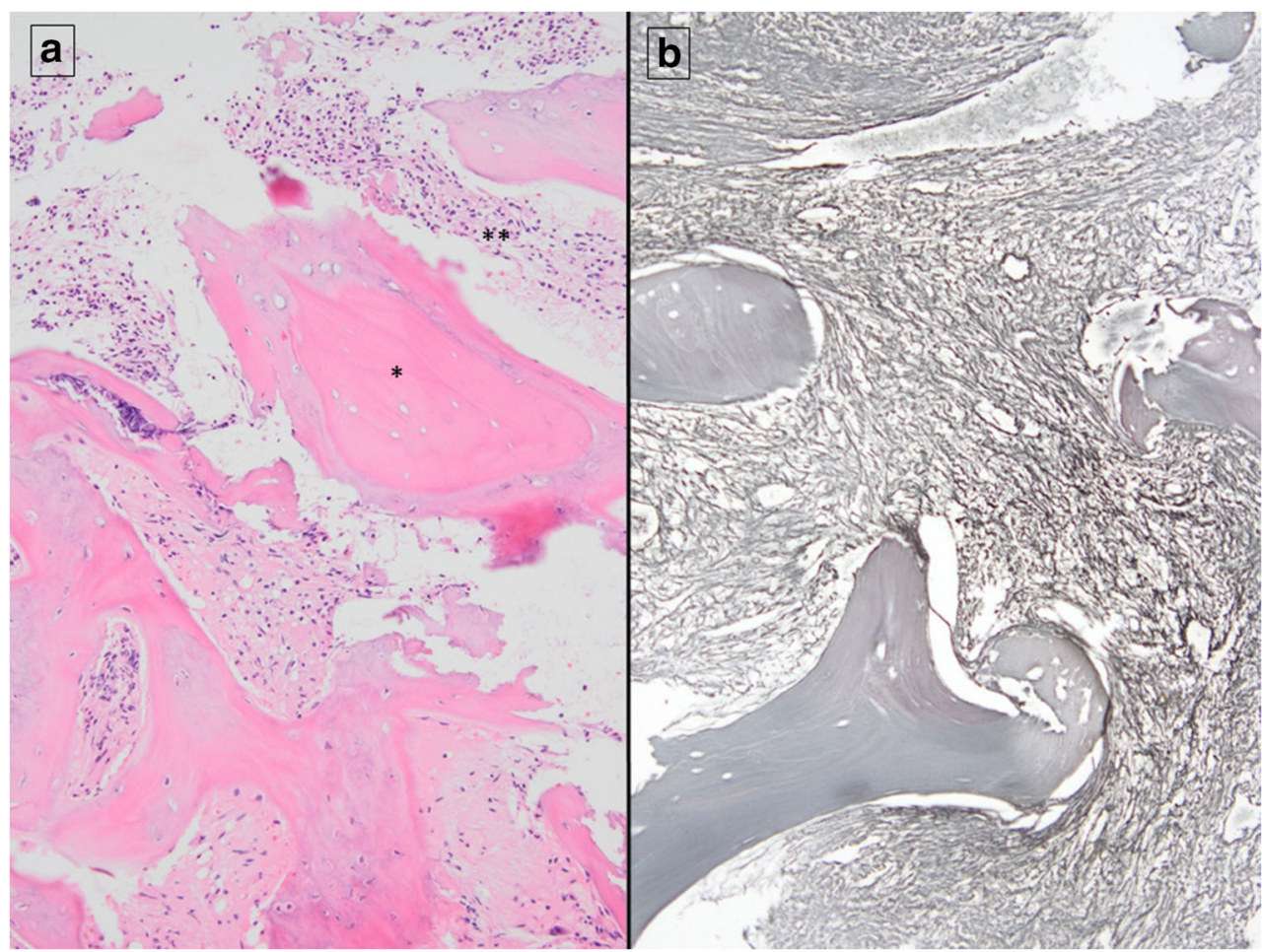




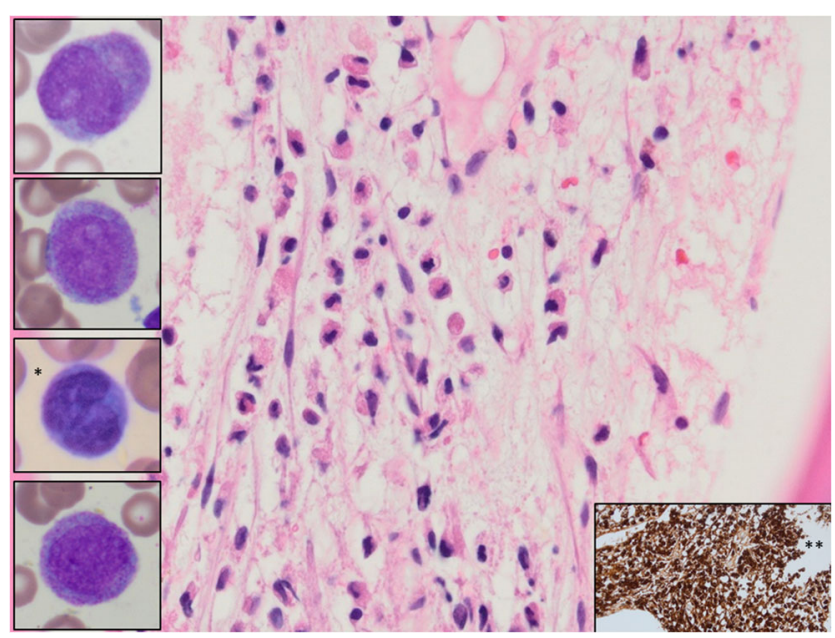

Fig. 4 High power magnification of the cellular area in the bone marrow biopsy shows immature cells with eccentric reniform nuclei and abundant eosinophilic cytoplasm, resembling myeloid precursors. Insets on the left hand show that May-Grünwald-Giemsa stain of the aspirate shows scant blastic cells with prominent nucleoli, note bilobed nucleus of one of these cells $(*)$. The inset on the bottom right shows myeloperoxidase positivity $(* *)$

cytokines in our patient. Similar to our case, a report described a 28-year-old female patient who had a slow-evolving APL with severe reticulin fibrosis [5]. Additionally, our case showed osteosclerosis that we presume was associated with the MF accompanying the APL. A study reported, $7.4 \%$ of children with leukemia had osteosclerosis at presentation [7]. They concluded that osteosclerosis developed from the reactive new bone formation due to leukemic cell infiltration and osseous infarction.

The prognostic importance of increased reticulin fibrosis associated with AML subtypes is not well elucidated. Studies on adult patients suffering from various disorders show a worse prognosis in the presence of MF. Lower grades of bone marrow fibrosis were associated with better survival in patients with primary MF [8]. Buesche et al. showed that bone marrow fibrosis in chronic myeloid leukemia patients during imatinib treatment was a predictor of imatinib failure, the risk of progression to accelerated phase, and mortality [9]. Additionally, bone marrow fibrosis grade 2 or higher is a poor prognostic factor in patients with MDS [10]. Primary AML patients with bone marrow fibrosis at diagnosis had earlier relapse and shorter survival rates [11]. A study has revealed that high reticulin fiber density on bone marrow was an unfavorable prognostic factor in childhood ALL patients as well [12]. On the contrary, Losada et al. reported a case series

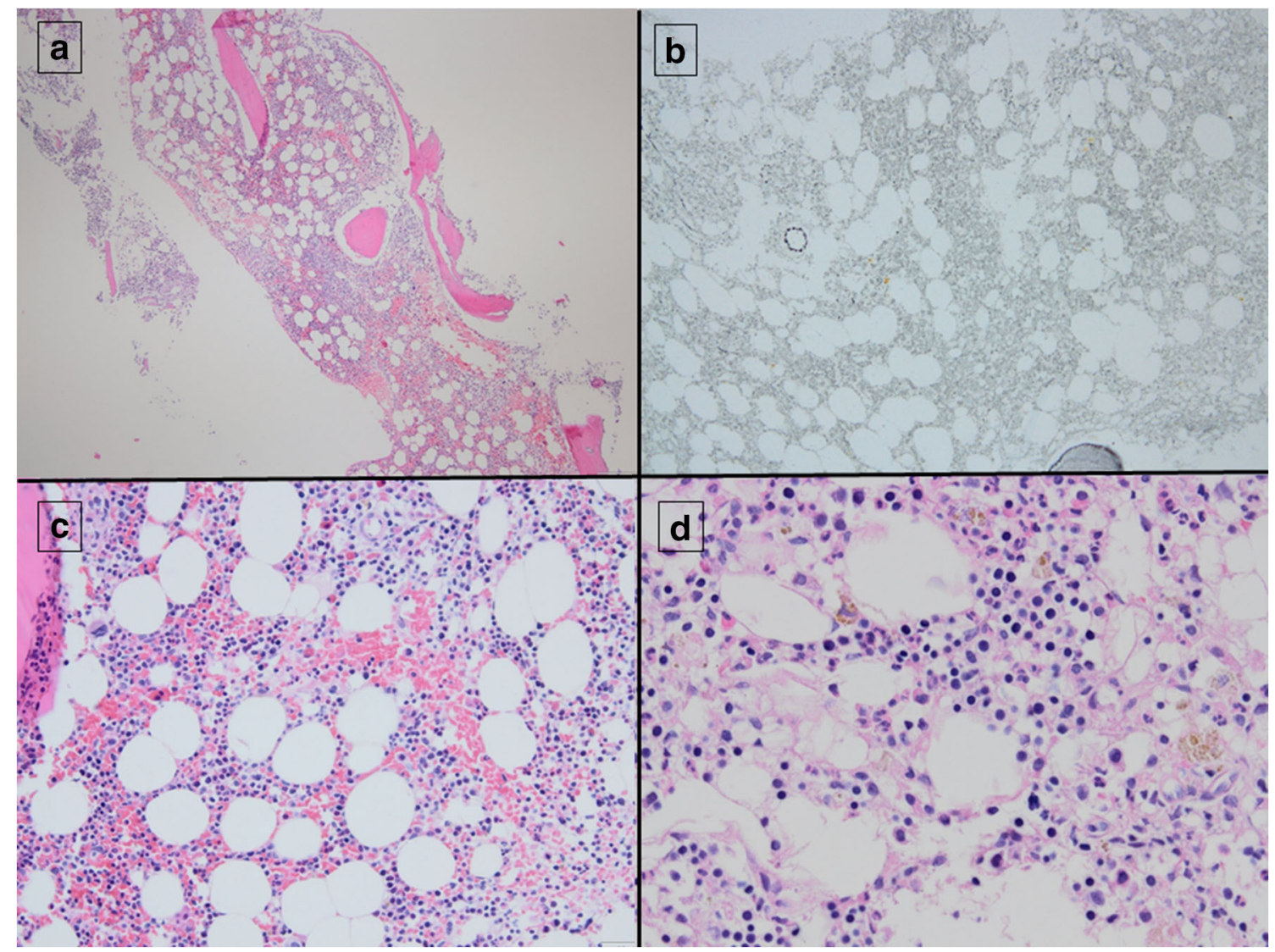

Fig. 5 Photomicrographs of the post-treatment follow-up bone marrow biopsy. Low power magnification reveals a slightly hypocellular bone marrow (A) with no visible reticulosis, which is confirmed with the reticulin stain (B). Higher power magnifications show preserved hematopoietic activity and maturation $(\mathbf{C}, \mathbf{D})$ 
including 12 children and 39 adult patients with APL, seven of them (13.7\%) showed MF (two children and five adults), and severe MF was present in two patients (3.9\%). They concluded that no significant difference in the prognosis of APL patients was observed in terms of the presence or absence of MF [13]. Case reports concerning MF in APL documented similar remission rates as in APL cases without MF [5, 6, 14-18]. According to these reports, MF in APL patients was not associated with an inferior outcome. Additionally, bone marrow fibrosis was reversible in some of the patients with APL, as in our case $[5,6,15,16]$. Currently, our patient is in morphological and molecular remission and has completed anti-leukemic treatment. BFM-AML 2013 protocol recommends ATRA therapy with cytotoxic chemotherapy. However, the BFM group recommends ATRA and arsenic trioxide combination in the contemporary 2019 protocol while omitting cytotoxic chemotherapy in low-risk (initial leukocyte count $<10 \times 10^{9}$ / L) APL patients.

The case highlights that fibrosis in the marrow can make it more challenging to diagnose acute leukemia. PML-RARA transcripts can be present with subtle clinical or hematological findings, which emphasize the importance of molecular studies in unexplained cytopenias. Acute promyelocytic leukemia should be considered in the differential diagnosis of the patients with cytopenias plus hypofibrinogenemia. Also, in the case of APL, as opposed to other myeloid neoplasms, fibrosis does not appear to be associated with a worse prognosis and can be resolved after treatment.

\section{Compliance with ethical standards}

Conflict of interest The authors declare that they have no conflict of interest.

Informed consent The consent of the parents of the patient is included in the hospital clinical documents.

\section{References}

1. Kuter DJ, Bain B, Mufti G, Bagg A, Hasserjian RP (2007) Bone marrow fibrosis: pathophysiology and clinical significance of increased bone marrow stromal fibres. Br J Haematol 139(3):351362

2. Bae E, Park CJ, Cho YU, Seo EJ, Chi HS, Jang S, Lee KH, Lee JH, Lee JH, Suh JJ, Im HJ (2013) Differential diagnosis of myelofibrosis based on WHO 2008 criteria: acute panmyelosis with myelofibrosis, acute megakaryoblastic leukemia with myelofibrosis, primary myelofibrosis and myelodysplastic syndrome with myelofibrosis. Int J Lab Hematol 35(6):629-636

3. Conneely SE, Stevens AM (2020) Advances in pediatric acute promyelocytic leukemia. Children (Basel) 7(2):11

4. Aksu T, Fettah A, Bozkaya İ, Baştemur M, Kara A, Çulha VK, Özbek NY, Yaralı N (2018) Acute promyelocytic leukemia in children: a single centre experience from Turkey. Mediterr J Hematol Infect Dis 10(1):e2018045

5. Abou Dalle I, Nassif S, Bazarbachi A (2018) Acute promyelocytic leukemia with increased bone marrow reticulin fibrosis: description of three cases and review of the literature. Hematol Oncol Stem Cell Ther 11(2):99-104

6. Mori A, Wada H, Okada M, Takatsuka H, Tamura A, Fujimori Y, Okamoto T, Takemoto Y, Kanamaru A, Kakishita E (2000) Acute promyelocytic leukemia with marrow fibrosis at initial presentation: possible involvement of transforming growth factor-beta(1). Acta Haematol 103(4):220-223

7. Sinigaglia R, Gigante C, Bisinella G, Varotto S, Zanesco L, Turra S (2008) Musculoskeletal manifestations in pediatric acute leukemia. J Pediatr Orthop 28(1):20-28

8. Gianelli U, Vener C, Bossi A, Cortinovis I, Iurlo A, Fracchiolla NS, Savi F, Moro A, Grifoni F, De Philippis C, Radice T, Bosari S, Lambertenghi Deliliers G, Cortelezzi A (2012) The European consensus on grading of bone marrow fibrosis allows a better prognostication of patients with primary myelofibrosis. Mod Pathol 25(9): 1193-1202

9. Buesche G, Ganser A, Schlegelberger B, von Neuhoff N, Gadzicki D, Hecker H, Bock O, Frye B, Kreipe H (2007) Marrow fibrosis and its relevance during imatinib treatment of chronic myeloid leukemia. Leukemia 21(12):2420-2427

10. Ramos F, Robledo C, Izquierdo-García FM, Spanish Group for Myelodysplastic Syndromes (GESMD) et al (2016) Bone marrow fibrosis in myelodysplastic syndromes: a prospective evaluation including mutational analysis. Oncotarget 7(21):30492-30503

11. Wu Z, Chen R, Wu L, Zou L, Ding F, Wang M, Liu X (2017) Bone marrow fibrosis at diagnosis predicts survival for primary acute myeloid leukemia. Clin Transl Oncol 19(12):1462-1468

12. Norén-Nyström U, Roos G, Bergh A, Botling J, Lönnerholm G, Porwit A, Heyman M, Forestier E (2008) Bone marrow fibrosis in childhood acute lymphoblastic leukemia correlates to biological factors, treatment response and outcome. Leukemia 22(3):504 510. https://doi.org/10.1038/sj.leu.2405072

13. Losada R, Cabrera H, Hernández $\mathrm{P}$, Hernández C, Menéndez A, Mesa J, Plascencia A, Ramón L, Agramonte O, Espinosa E (2002) Bone marrow reticulin fibrosis at diagnosis in promyelocytic leukaemia treated with all-trans retinoic acid has no adverse prognosis. Acta Haematol 108(2):111-112

14. Shah H, Bradford C, Sayar H (2015) Acute promyelocytic leukemia presenting with severe marrow fibrosis. Case Rep Hematol 2015: 826894

15. Lu Q, Chen Y, Li Z (2012) Long-term remission in a case of acute promyelocytic leukemia patient with marked myelofibrosis treated with arsenic trioxide, all-trans retinoic acid and consolidation therapy with daunorubicin plus cytarabine. Leuk Res 36(6):e119-e121

16. Fukuno K, Tsurumi H, Yoshikawa T, Yamada T, Oyama M, Moriwaki H (2001) A variant form of acute promyelocytic leukemia with marked myelofibrosis. Int J Hematol 74(3):322-326

17. Dutta P, Hasan S, Bhattacharyya J, Kumar R, Mahapatra M, Saxena R, Tyagi S, Sazawal S, Pati HP (2006) Acute promyelocytic leukemia with secondary myelofibrosis - case report and review of the literature. Am J Hematol 81(6):476-477

18. Aventín A, Mateu R, Martino R, Colomer D, Bordes R (1998) A case of cryptic acute promyelocytic leukemia. Leukemia 12(9): 1490-1491

Publisher's note Springer Nature remains neutral with regard to jurisdictional claims in published maps and institutional affiliations. 